\title{
CHAMP observation of intense kilometer-scale field-aligned currents, evidence for an ionospheric Alfvén resonator
}

\author{
M. Rother ${ }^{1}$, K. Schlegel ${ }^{2}$, and H. Lühr ${ }^{1}$ \\ ${ }^{1}$ GeoForschungsZentrum Potsdam, Germany \\ ${ }^{2}$ Max-Planck-Institute for Solar System Research (formerly MPAE Lindau), Katlenburg-Lindau, Germany
}

Received: 8 December 2006 - Revised: 7 June 2007 - Accepted: 27 June 2007 - Published: 30 July 2007

\begin{abstract}
Bursts of very intense kilometer-scale fieldaligned currents (KSFACs) are observed quite frequently by the CHAMP satellite when passing through the auroral region. In extreme cases estimated current densities exceed $3 \mathrm{~mA} / \mathrm{m}^{2}$. Typical scale sizes of these KSFACs are $1 \mathrm{~km}$.
\end{abstract}

The low-Earth, polar orbiting satellite CHAMP allows one to assess KSFACs down to scales of a couple of $100 \mathrm{~m}$ based on its high-precision magnetic field vector data sampled at $50 \mathrm{~Hz}$. Using data from 5 years (2001-2005) details of these currents can be investigated. In our statistical study we find that most of the KSFAC bursts and the strongest events are encountered in the cusp/cleft region. Significantly fewer events are found on the nightside. The affected region is typically $15^{\circ}-20^{\circ}$ wide in latitude. There seems to be some dependence of the current intensity on the level of magnetic activity, $K_{p}$. On the other hand, no dependence has been found on sunspot number, the solar flux level, F10.7 or the solar zenith angle. The latitude, at which KSFAC bursts are encountered, expands equatorward with increasing $K_{p}$. This trend follows well the auroral oval expansion during enhanced magnetic activity. These KSFACs are generally accompanying large-scale FAC sheets, and they are predominantly associated with Region 1 currents. We propose an explanation of the KSFACs in terms of Alfvén waves trapped in a ionospheric resonator, which is initiated when the convection electric field or current strength surpasses a critical value. Many properties of such a resonator are in agreement with our KSFAC results.

Keywords. Ionosphere (Auroral ionosphere; Ionospheremagnetosphere interactions) - Magnetospheric physics (MHD waves and instabilities)

Correspondence to: $\mathrm{M}$. Rother

(rother@gfz-potsdam.de)

\section{Introduction}

Field-aligned currents (FACs) in the auroral zone are observed over a wide range of spatial scales covering wavelengths from $1 \mathrm{~km}$ to several $100 \mathrm{~km}$. There is a general FAC characteristic, the shorter the wavelength the larger the current density (e.g. Ritter and Lühr, 2006, Fig. 10). In particular, small-scale field-aligned currents have been a subject of growing interest in recent years. After their first detection with instruments on board the DE satellites (Burch et al., 1983) and the Viking satellite (Potemra et al., 1987) their importance for the plasma dynamics has been realized. At that time it was not possible, however, to identify the smallest scales carrying the highest currents. The resolution of spatial scale has been continuously reduced to some ten kilometers achieved by sounding rockets (Lühr et al., 1987) and the Akebono spacecraft (Fukunishi et al., 1991). Finally a few $100 \mathrm{~m}$ could be reached with the Freja and Ørsted satellites (Lühr et al., 1994; Neubert and Christiansen, 2003) and also with the CHAMP satellite, as presented here. Simultaneously, the peak amplitudes of the current density of these KSFACs has been found to increase from tens of $\mu \mathrm{A} / \mathrm{m}^{2}$ to more than $1 \mathrm{~mA} / \mathrm{m}^{2}$, with decreasing scale length.

Small-scale FACs are embedded within the large-scale current regions (Region 1 and Region 2, as defined by Iijima and Potemra (1976) but also in the cusp and polar cap current systems (Fukunishi et al., 1991; Neubert and Christiansen, 2003). Golovchanskaya et al. (2006) examined DE2 data and found that most intense small-scale electromagnetic turbulence is associated with enhanced large-scale FACs. The highest current densities have been observed near the cusp region (Neubert and Christiansen, 2003).

The term small-scale FAC has been used in the past for different ranges of wavelengths, primarily depending on the resolution of the analysed data. For this study we would like to introduce the FAC classification "large-scale" for transverse wavelengths of more than $100 \mathrm{~km}$, "medium-scale" for

Published by Copernicus Publications on behalf of the European Geosciences Union. 
$100-5 \mathrm{~km}$ and "kilometer-scale" for the range $5 \mathrm{~km}$ to below $1 \mathrm{~km}$. Here we will focus on the latter scale and use subsequently the abbreviation KSFAC. Due to instrumental limitations, not many observations of the magnetic signatures of these small structures have been reported. The study by Fukunishi et al. (1991), for example, based on Akebono data belongs, according to our classification, to mediumscale FACs. There is one extensive survey of KSFACs in the auroral region derived from Ørsted measurements (Neubert and Christiansen, 2003). They established a clear preference of these intense FAC structures on the dayside, and mentioned enhanced occurrence rates on the nightside during magnetically active periods. Several attempts to correlate the observed FAC densities or occurrence frequencies with solar wind conditions were not successful. The authors offer no clear explanation for the generation mechanism.

These KSFACs are probably linked to Alfvén waves with small transverse wavelengths. In several cases electric field measurements have been used to study their features, Boehm et al. (1990), for instance, found amplitudes of $E>100 \mathrm{mV} / \mathrm{m}$ from high-resolution rocket measurements above $700 \mathrm{~km}$. Chmyrev et al. (1985) reported a maximal value of $\pm 200 \mathrm{mV} / \mathrm{m}$ observed with the Intercosmos BULGARIA-1300 satellite at $811 \mathrm{~km}$. Multi-scale current structures with amplitudes of $100-300 \mu \mathrm{A} / \mathrm{m}^{2}$ at subkilometer scales examined with the Freja satellite have been linked to interference patterns of inertial Alfvén waves by Stasiewicz and Potemra (1998). A rather comprehensive study of inertial Alfvén waves based on Akebono observations in the cusp topside ionosphere was presented by Hirano et al. (2005). These authors give many references to similar studies derived from measurements of other spacecraft. They investigated the wave characters at three different altitudes in the range $8000 \mathrm{~km}$ to $1500 \mathrm{~km}$. At the lowest level they find clear evidence for short wavelength Alfvén waves trapped in the ionospheric Alfvén resonator (IAR).

One of the first theoretical investigations proposing an IAR are those by Trakhtengertz and Feldstein (1984); Trakhtengerts and Feldstein (1991) and Lysak (1991). The IAR is bounded from below by the ionospheric $\mathrm{E}$ layer and from above by a broad zone of rapidly increasing Alfvén velocity, due to the decreasing plasma concentration above the Flayer peak up to $10^{4} \mathrm{~km}$ height. The Alfvén waves trapped in the resonator and powered by the magnetospheric convection ultimately cause turbulent plasma processes, resulting in the formation of a turbulent Alfvén boundary layer (Trakhtengerts and Feldstein, 1991). Lysak (1991) deduced from model calculations that there exists a distinct phase relation between the E- and B-fields of the waves in the IAR. Such a relation has been experimentally identified for small-scale waves (Dubinin et al., 1985; Hirano et al., 2005). A somewhat modified Alfvén resonator has been recently proposed by Pilipenko et al. (2002) using results from the reflection and transmission of waves at the auroral acceleration region (AAR) derived by Vogt and Haerendel (1998). Contrary to the common IAR, where the upper reflection boundary is the strong gradient of the Alfvén velocity above the F-layer maximum, the upper reflection boundary of this resonator is assumed to be the AAR. This region is assumed to be located much higher, i.e. at about $1 R_{E}$. The bottom side boundary of this resonator associated with the AAR (or RAAR, as called by Pilipenko et al., 2002) is the ionospheric E-layer (as it is also for the common IAR). The reflection conditions on both sides depend on the wavelength, as well as on the conductivities of the E-layer and the auroral acceleration region. The lowest eigenfrequency of the RAAR is about $0.1 \mathrm{~Hz}$, the transverse scales are a few kilometers (Pilipenko et al., 2002).

So far, there are no global surveys of the spatial distribution and the temporal occurrence rates of an Alfvén resonator. It has not been experimentally verified what the typical conditions are which accompany the resonator, and which wave amplitudes can develop within it. In Sect. 5 we propose an explanation of the observed KSFACs in terms of the RAAR. Many details of the corresponding waves, as well as the generation processes for the KSFACs are still poorly understood. We think that some of these open issues can be addressed with the high-resolution magnetic field measurement of the CHAMP satellite. Although we have no suitable electric field measurement, we think, the magnetic field data can efficiently be used for this purpose. This is, in particular, true at the low cruising altitude of $400 \mathrm{~km}$, where the magnetic signature of resonant waves is about to maximise and the E-field to disappear. The long and continuous data series of CHAMP, covering all longitudes and local times, is a good basis for a comprehensive survey of KSFACs in terms of this resonance phenomenon.

\section{Instrumentation and data processing}

The satellite CHAMP (Reigber et al., 2002) was launched on 15 July 2000 into a circular, near-polar $\left(87.3^{\circ}\right.$ incl.) orbit. From its initial altitude at $456 \mathrm{~km}$ the orbit has decayed to about $350 \mathrm{~km}$ after 5 years. The orbital plane precesses at a rate of $1 \mathrm{~h}$ in local time (LT) per 11 days, thus visiting all local times within 131 days. The time period covered for this study lasts from January 2001 to September 2005, which provides a 13 -fold coverage of all local times.

The data interpreted here are the vector magnetic field measurements of the Fluxgate Magnetometer (FGM). This instrument delivers vector field readings at a rate of $50 \mathrm{~Hz}$ and a resolution of $0.1 \mathrm{nT}$. The data are calibrated routinely with respect to the onboard absolute scalar Overhauser Magnetometer (OVM). This ensures a continuously high data quality. A dual-head star camera system mounted together with the FGM on an optical bench provides the orientation of the measured field vector with arc-second precision. Data used for this study are transformed into a Mean-FieldAligned (MFA) coordinate system. In this local frame the $\mathrm{z}$-axis is aligned with the average field direction, the $\mathrm{y}$-axis 
is perpendicular to the magnetic meridian, pointing eastward, and the $\mathrm{x}$-axis completes the triad.

For the transformation of the field vectors into the MFA frame we did not take the individual readings of the star camera, since it would add a significant amount of noise to the FAC time series. Instead, we have determined the mean field from an average of the measured field vector over a period of $60 \mathrm{~s}$ (corresponding to $440 \mathrm{~km}$ along track).

For the estimate of the FAC density the simple 1-D approach was used where recorded temporal variations are interpreted as spatial structures. Since FACs generate only transverse field disturbances, we have used the data in MFA coordinates.

For the actual calculation of the FAC density, we used the reduced Ampère's law

$j_{z}=\frac{1}{\mu_{0} v_{\perp}} \frac{\Delta B_{y}}{\Delta t}$,

where $v_{\perp}$ is the velocity component which is perpendicular to the magnetic field and in the orbital plane, $\Delta B_{y}$ is the difference between consecutive readings of the MFA $B_{y}$ component, and $\Delta t=20 \mathrm{~ms}$ is the time step of measurements. We are aware that the KSFACs are not well organised in current sheets. This will result in an underestimation of the current density and an overestimation of the equivalent scale, as was shown by Lühr et al. (1996), even though there seems to be a certain tendency to form elongated current sheet structures, as determined from a minimum variance analysis (not shown). The ratio of the largest to the medium eigenvalue derived from the two perpendicular field components is only of the order of 2. Furthermore, these small-scale FACs are expected to be rather dynamic. Our assumed stationarity may thus not be valid. From ground observations Belyaev et al. (1999) reported frequencies for the ionospheric Alfvén resonator of 0.5 to $1.5 \mathrm{~Hz}$. Due to the small transverse scale size $(\approx 1 \mathrm{~km})$ and the high orbital velocity $(7.6 \mathrm{~km} / \mathrm{s})$, the crossing of the considered current structure takes only $0.1-0.2 \mathrm{~s}$. During this time the wave phase cannot change too much. The temporal variation will, in some cases, enhance the resulting current density, and in other cases reduce it. What we obtain may be called an "equivalent FAC density" (subsequently shortened to FAC). We assume, however, that the effects average out statistically.

The resolution of the obtained current density is limited by the digitisation steps. A field change of $0.1 \mathrm{nT}$ in $20 \mathrm{~ms}$ converts to a FAC density step of $\Delta j_{z} \approx 0.8 \mu \mathrm{A} / \mathrm{m}^{2}$. This is very small compared to the amplitudes considered here. At this point it should be noted that the subsequent study focuses on the characteristics of intense KSFACs. The absolute accuracy of the estimated FAC density is therefore of less concern for the conclusions.

Besides the current densities obtained with Eq. (1) from the $50-\mathrm{Hz}$ resolution magnetometer readings we calculated a corresponding condensed data set with a $1-\mathrm{Hz}$ resolution. To obtain this we computed the root mean squared $50-\mathrm{Hz}$

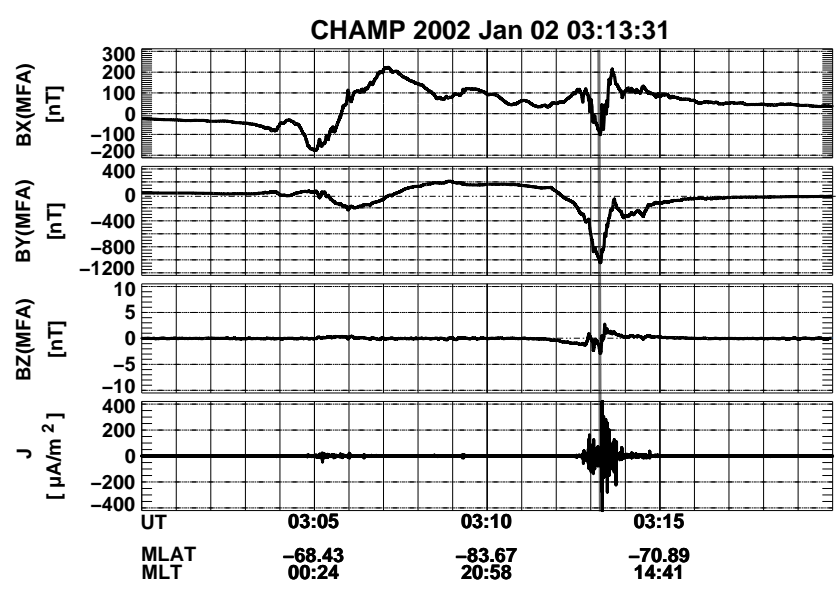

Fig. 1. Example case of high resolution CHAMP Fluxgate Magnetometer data in a mean field-aligned coordinate system (upper three panels) and a derived small-scale field-aligned current density (lowest panel).

current densities values over $1 \mathrm{~s}$. By this procedure we conserved the energy of the KSFACs. This condensed data set is referred to as $1-\mathrm{Hz}$ data in the following.

\section{Typical equivalent FAC characteristics}

In this section we introduce the typical features of the KSFACs. Special emphasis will be put on the peak amplitude of current density observed within a burst. Since the satellite moves through the KSFAC with a high velocity $(7.6 \mathrm{~km} / \mathrm{s})$, we consider the current estimates based on the high-resolution $50-\mathrm{Hz}$ data as spatial features of these extreme current filaments.

Figure 1 shows an example of the magnetometer data during a pass over the Southern Hemisphere auroral region. In the upper three panels the magnetic field vector components are displayed, rotated into the MFA coordinate system. The lowest panel shows the field-aligned current density calculated from the corresponding $50-\mathrm{Hz}$ magnetometer readings. The KSFAC bursts occur within the auroral current regions, with the typical behaviour of the dayside currents (around 12:00 MLT) being considerably stronger than the night side currents (around 00:00 MLT).

In Fig. 2 the time scale of a similar burst is enlarged, showing details around the peaks of the currents. It is obvious that the temporal width of these large peaks and their separation are well resolved by the time resolution $(0.02 \mathrm{~s})$ of the instrument. The equivalent scale drawn in the figure indicates that the width of a major peak is typically of the order of $1 \mathrm{~km}$. We examined this spatial scale more quantitatively by calculating the temporal spectra of the KSFAC bursts. Figure 3 shows a single spectrum and an average of many spectra. It can be separated into two regions: at lower frequencies it is 

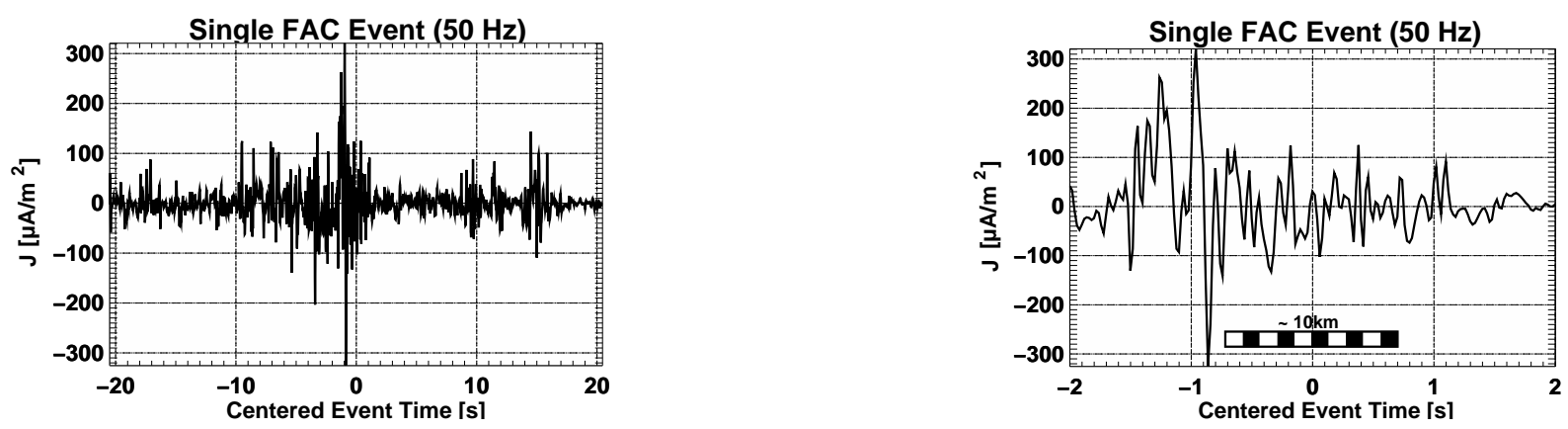

Fig. 2. Example of KSFAC burst event (left) and enlarged detail at 50-Hz resolution (right).

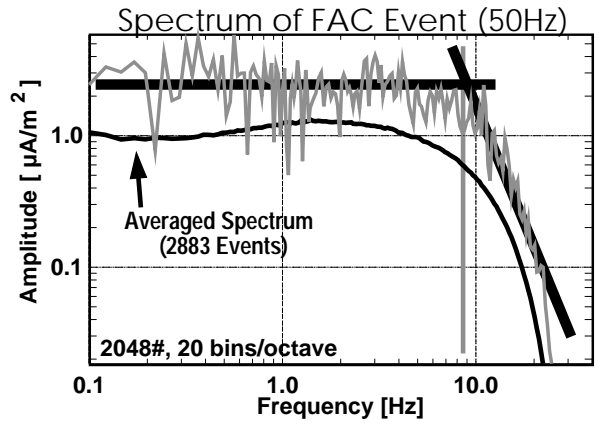

Fig. 3. Single event and average spectrum of $50-\mathrm{Hz}$ current bursts.

practically flat, and at high frequencies it decreases sharply. A fit to this behaviour (with a power law at high frequencies) reveals a "cut-off frequency" of about $8 \mathrm{~Hz}$. With the average spacecraft velocity of about $7.6 \mathrm{~km} / \mathrm{s}$ this corresponds to a "cut-off" wavelength of about $1 \mathrm{~km}$, in agreement with the visual inspection. This number is in agreement with earlier findings from the Freja satellite (Lühr et al., 1994). When looking at many KSFAC bursts and calculating the average spectrum the main features are still the same, such as the flat, low frequency part, and a decrease at frequencies beyond $10 \mathrm{~Hz}$ occurs, the transition is, however, somewhat smoother. This means that the typical scale size may differ somewhat from event to event but only within a limited range.

In the following we will examine these KSFAC bursts in detail and determine some of their statistical properties. In order to do this we have to establish a list of considered "events", as they are called in the following. This is not straightforward since they are quite numerous, in general, several bursts are observed per orbit. In order to reduce the data to a manageable quantity, we worked with the condensed data set of $1-\mathrm{Hz}$ resolution.

As a selection criterion we require in a first step a peak current density of $|j|>30 \mu \mathrm{A} / \mathrm{m}^{2}$. In a next step, those peaks exceeding the threshold and being not too far separated from each other, are grouped together in a burst. Peaks are recog-
J 1Hz 2002-06-05

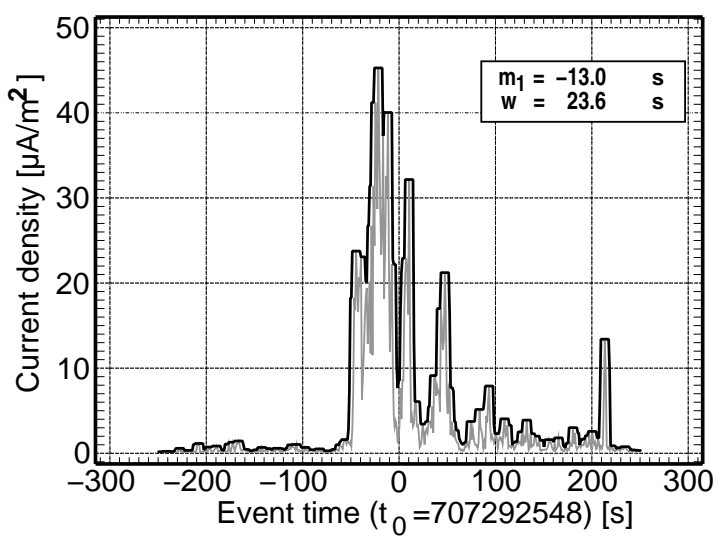

Fig. 4. Example of event at $1-\mathrm{Hz}$ resolution, together with moments for characterisation of a KSFAC event.

nised as part of the same burst if a separation of $30 \mathrm{~s}$ to the neighbouring structure is not exceeded. This period, chosen after extensive testing, is close to a typical length of a burst event itself (see later Fig. 13). In addition, we restricted ourselves to events at high latitudes, $|\mathrm{MLAT}|>50^{\circ}$, since a preliminary overview revealed that only very few events are observed at lower latitudes.

The established event table has 21417 entries. Since our total database comprises the time interval of 1 January 2001 to 6 September 2005, we examined 4.68 years. The total number of CHAMP orbits during this interval is $26600 \approx 4.68 \times 365 \times 15.6$, which means 106400 crossings of the auroral zone (four per orbit). Thus, our event table contains, on average, one event per five auroral zone crossings. It should be noted, however, that much more events are identified if we reduce the above threshold level of $|j|>30 \mu \mathrm{A} / \mathrm{m}^{2}$.

In a next step we have to quantify the characteristics of an KSFAC event. These are explained with the help of Fig. 4. It shows as a thin grey line the temporal structure of an event, 
i.e. the $1-\mathrm{Hz}$ absolute value of the current density versus time. This time series is then characterised by a staircase envelope with a step length of $10 \mathrm{~s}$ (black line). From this envelop time series $\left(j_{1 \mathrm{~Hz}, \mathrm{e}}\right)$ the first two moments are calculated to characterise the shape of the events:

1. Its occurrence time: to localise the event in the satellites time frame $t$,

$$
m_{1}=\sum\left(t j_{e}\right) / \sum j_{e}
$$

2. The distribution width $w_{1}$ of the event, represented by the square root of the variance:

$$
w=\left(\sum\left(t^{\prime 2} j_{e}\right) / \sum j_{e}\right)^{1 / 2}
$$

where $t^{\prime}=t-m_{1}$.

We tried several other ways to characterise our KSFAC events, applying various degrees of smoothing or fitting to distribution functions. According to our studies, the method described above yields the most convincing quantification of the events. Note that this kind of envelope is often applied in establishing geophysical quantities (e.g. $A E, K_{p}$ ).

\subsection{Occurrence frequency of KSFAC events}

At first we looked at the occurrence frequency of the observed KSFAC events. Figure 5a shows this frequency separated by seasons and by day/night. It is quite obvious that KSFAC events occur predominantly during sunlit conditions: generally, they are more frequent during the day than during night, and considerably more in summer than in winter. The equinox-night value stands out somewhat of this scheme; it is higher than the corresponding summer night value. This is probably a consequence of the geomagnetic activity peaking at equinox. We will come back to this in the Discussion section.

Figure 5b shows the corresponding occurrence frequencies of the different years of our observation interval. The frequency follows the magnetic activity variations over the solar cycle. The annual averages of both the $A_{p}$ index and the KSFAC occurrence frequency peak at 2003 and decline towards solar activity minimum. We will offer an explanation for this behaviour in Sect. 5 .

\subsection{Relationship between j-50 Hz Peaks and j-1 Hz Peaks}

As mentioned before, the event selection and characterisation is based on the $1-\mathrm{Hz}$ data. We nevertheless are interested in the fine structure of the events. To enable this the maximum

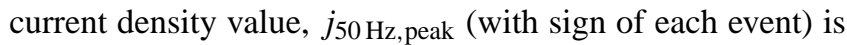
also included in our event table. This peak is generally larger than the peak of the $1-\mathrm{Hz}$ data because the latter is subject to averaging. Figure 6 shows a scatter plot of $1-\mathrm{Hz}$ (peaks) versus $50-\mathrm{Hz}$ current density peaks.
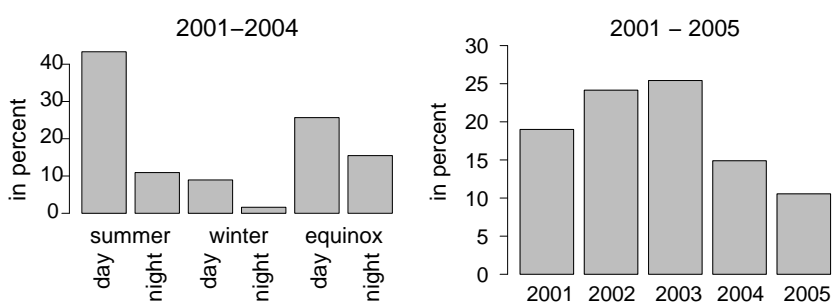

Fig. 5. Occurrence frequency of KSFAC events as a function of season and day/night (a), and as a function of time during our observation interval (b). The numbers are normalised to the number of auroral zone crossings.

MLT Day: Circle, MLT Night: Square All: 3.75 , Day: 3.76 , Night: 3.69

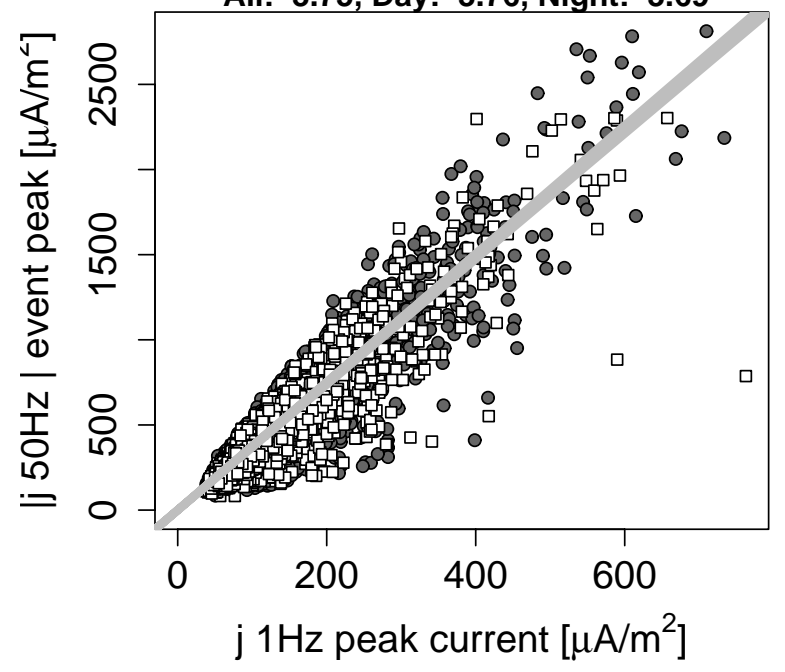

Fig. 6. Correlation of $\left|j_{50 \mathrm{~Hz} \text {,peak }}\right|$ versus $j_{1 \mathrm{~Hz}, \max }$. The slopes of the regression lines are given on top of the figure for various selections.

We have separated the event database into daytime (05:0017:00 MLT) and nighttime (17:00-05:00 MLT) events. Although there is some scatter, the difference between the indicated regression line (slopes in the legend on top of the figure) is statistically insignificant, and it indicate that - practi-

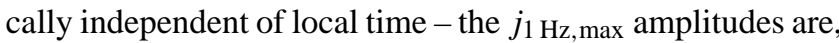
on average, about a factor of $3-4$ lower than the $\mid j_{50 \mathrm{~Hz}}$,peak $\mid$ amplitudes. This ratio can be regarded as a kind of crest factor of the $50-\mathrm{Hz}$ peaks compared to the neighbouring FAC densities. We want to stress here that we use both quantities to characterise our events in the following. The $50-\mathrm{Hz}$ current peak gives an unprocessed estimate of the largest finestructure current density within a burst; the $j_{1 \mathrm{~Hz} \text {,max }}$ is more representative for the average current density of the whole burst. 

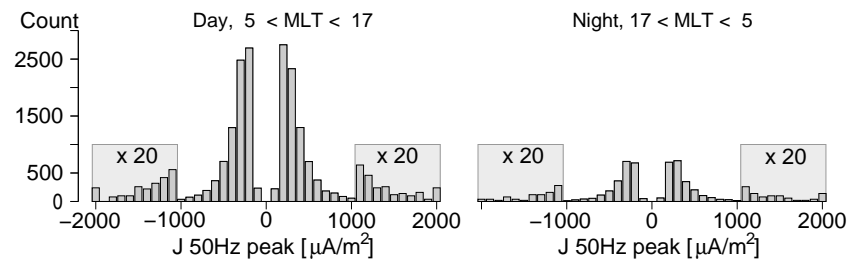

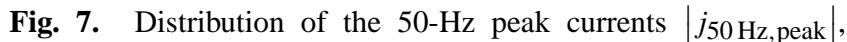
separated for daytime and nighttime. Events with $\left|j_{50 \mathrm{~Hz}, \text { peak }}\right|>2000 \mu \mathrm{A} / \mathrm{m}^{2}$ are grouped together in one bin. Bin heights for $\mid j_{50 \mathrm{~Hz}}$,peak $\mid>1000 \mu \mathrm{A} / \mathrm{m}^{2}$ are magnified by a factor of 20 .

\section{Statistical properties of KSFAC events}

\section{$4.150-\mathrm{Hz}$ current data}

The occurrence rate of the high resolution peak values $\mid j_{50 \mathrm{~Hz}}$,peak $\mid$ is shown in Fig. 7, separated for daytime and nighttime events. The distribution is rather symmetric in both cases. The number of positive values (upward currents) is approximately balanced by the number of negative values. The reduced occurrence towards the smallest values of current density is certainly a consequence of our threshold criteria. The maxima of the distribution, here at around $-250 \mu \mathrm{A} / \mathrm{m}^{2}$ and $+250 \mu \mathrm{A} / \mathrm{m}^{2}$, therefore depend on the threshold and are not preferential values. There is no significant difference in the distribution of the events between day and night, except for the absolute number of cases. Less than $1 / 3$ of the events in our event table are from nighttime (17:00-05:00 MLT). This is also evident in Fig. 8.

To demonstrate the relatively large number of events with high current densities, we have magnified the bar height for $\left|j_{50 \mathrm{~Hz} \text {,peak }}\right|>1000 \mu \mathrm{A} / \mathrm{m}^{2}$ by a factor of $20 \mathrm{in} \mathrm{Fig.} 7$. A certain number of events with very large current density peak values, $\left|j_{50 \mathrm{~Hz} \text {,peak }}\right|>2000 \mu \mathrm{A} / \mathrm{m}^{2}$, are observed (combined in the very last bin on each side in Fig. 7). We are convinced that these are not artifacts. In order to check this we inspected all events with $\left|j_{50 \mathrm{~Hz} \text {,peak }}\right|>2000 \mu \mathrm{A} / \mathrm{m}^{2}$, one by one individually. It turned out that the majority of these events occurred during highly disturbed conditions, for instance, during November 2001 or on 29-30 October 2003 (see also Wang et al., 2006). These high current amplitudes are therefore most probably real. Nevertheless, we set an upper limit, discarding all events with $\left|j_{50 \mathrm{~Hz} \text {,peak }}\right|>4000 \mu \mathrm{A} / \mathrm{m}^{2}$, in order to avoid spurious effects by data outliers.

The three events in our table with the highest peak amplitudes (after elimination of obvious artifacts) are $\left|j_{50 \mathrm{~Hz}, \text { peak }}\right|=3222,3076$, and $3311 \mu \mathrm{A} / \mathrm{m}^{2}$. These very large features are typically supported by 8 data points.

The distribution of KSFAC events in a magnetic latitude - magnetic local time frame is shown in Fig. 8 for two thresholds of $\left|j_{50 \mathrm{~Hz} \text {,peak }}\right|$ values $\left(300 \mu \mathrm{A} / \mathrm{m}^{2}\right.$ and

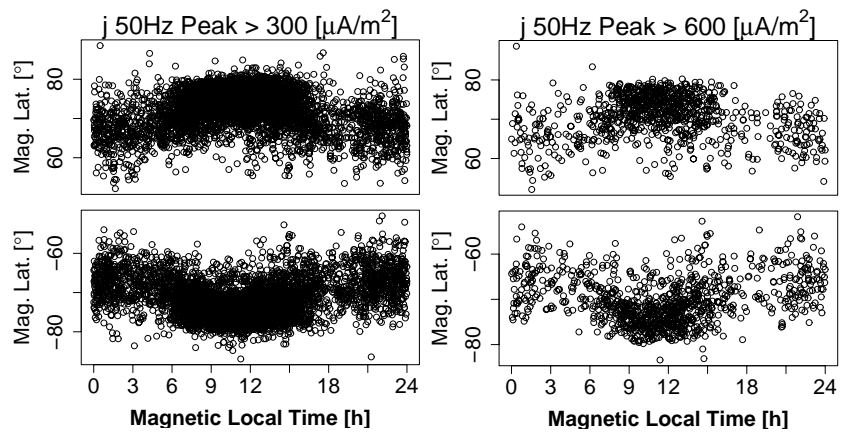

Fig. 8. Locations (in MLAT) of 50-Hz KSFAC peaks as a function of magnetic local time for two different threshold values.

Table 1. Median of peak current densities, $\left|j_{50 \mathrm{~Hz}, \text { peak }}\right|$ for different seasons (in $\mu \mathrm{A} / \mathrm{m}^{2}$ ).

\begin{tabular}{llll}
\hline & Summer & Winter & Equinox \\
months & $5,6,7,8$ & $1,2,11,12$ & $3,4,9,10$ \\
\hline day & 281 & 299 & 292 \\
night & 296 & 301 & 313 \\
\hline
\end{tabular}

$600 \mu \mathrm{A} / \mathrm{m}^{2}$ ). The majority of the events occur in a band of about $60^{\circ}<\mid$ MLAT $\mid<80^{\circ}$ which coincides practically with the auroral oval. Note that there is a clear concentration of the stronger events $\left(\left|j_{50 \mathrm{~Hz} \text {,peak }}\right|>600 \mu \mathrm{A} / \mathrm{m}^{2}\right)$ on the dayside. A closer inspection reveals that these stronger peaks are centred at about one hour before magnetic noon. The few strong events on the nightside occur during disturbed conditions (see also later Fig. 9). This tendency, as well as the grouping of events around 11 MLT, has been observed before (Neubert and Christiansen, 2003).

No significant variation of the $50-\mathrm{Hz}$ current density peaks on season was found. The median values of the $\left|j_{50 \mathrm{~Hz} \text {,peak }}\right|$ are hardly different for summer, winter and equinox, as Table 1 shows. Only the equinox nighttime value is slightly enhanced, which may be related to the well-known enhancement in geomagnetic activity during equinox seasons (Russell and McPherron, 1973).

The amplitude of the $50-\mathrm{Hz}$ current density peaks depends on geomagnetic conditions, as shown in Fig. 9, separately for day (left) and night (right). The median of the $\left|j_{50 \mathrm{~Hz} \text {,peak }}\right|$ values is indicated by the thick line, while their scatter within each $K_{p}$-bin is expressed by the quartiles (boxes) and the standard deviation indicated by dashed vertical lines. The increase in the current density median values with $K_{p}$ is quite moderate. During daytime it is somewhat stronger than during the night where the variation is insignificant. On the dayside the value is doubling between $K_{p} 0$ and 9 . Even though we see that the median of the peak current density increases 

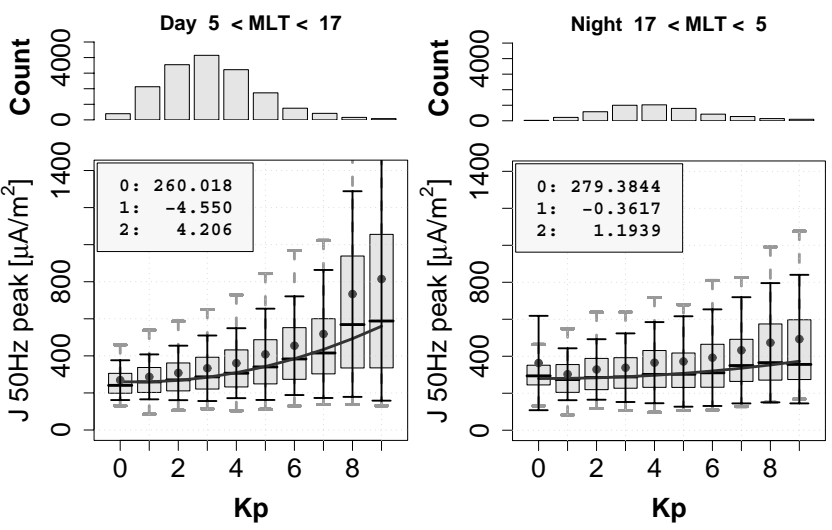

Fig. 9. $K_{p}$ dependence of the $\mid j_{50 \mathrm{~Hz}}$,peak $\mid$. The thick line within the box indicates the median value for the respective bin (the mean is marked by a small circle), the box reflects quartiles and the dashed vertical lines the standard deviation. The numbers in the legend denote the coefficients of the regression parabola.

with $K_{p}$ in a nonlinear fashion, we have fitted a degree 2 polynomial to the median values. The coefficients of a regression parabola are given in the figure legend. On top of the graphs there are frames containing information about the $K_{p}$-distribution of our events. Compared to the normal distribution of $K_{p}$ values our observed occurrence rate shows only a slight deviation. Details of this $K_{p}$ distribution will be discussed in the context of Fig. 11.

A clear relation exists between the observed latitude of $\left|j_{50 \mathrm{~Hz} \text {,peak }}\right|$ and $K_{p}$, as shown in Fig. 10. With an increase in the disturbance index the average locations move equatorward, which is in agreement with the expansion of the auroral oval during disturbed conditions. Again, the plotted regression parabola indicates a nonlinear behaviour of the relationship. It should be noted that other quantities have also been identified as influencing the latitudinal variation of FACs. In a previous study Wang et al. (2006) established a close control of the negative IMF $B_{z}$ amplitude on the latitudinal variation of the dayside large-scale FACs. In other cases the role of the solar wind dynamic pressure has been emphasised (Boudouridis et al., 2003). During low activity, $K_{p}=0$, KSFACs are found at $79^{\circ}$ and $70^{\circ}$ MLAT on the day- and nightside, respectively. For high magnetic activity the difference between the median latitudes decreases $\left(K_{p}=9\right.$, dayside: $63^{\circ}$, night side: $60^{\circ}$ ). This activity dependent latitude variation is comparable to that of the large-scale FACs (e.g. Wang et al., 2005a).

In order to obtain information about the distribution of the events in our table with respect to $K_{p}$, we prepared Fig. 11 . It shows the ratio of the normalised occurrence frequencies (for day $f^{\text {day }}$ and night $f^{\text {night }}$ in magnetic local time). The normalised occurrence frequency of the full set is calculated as
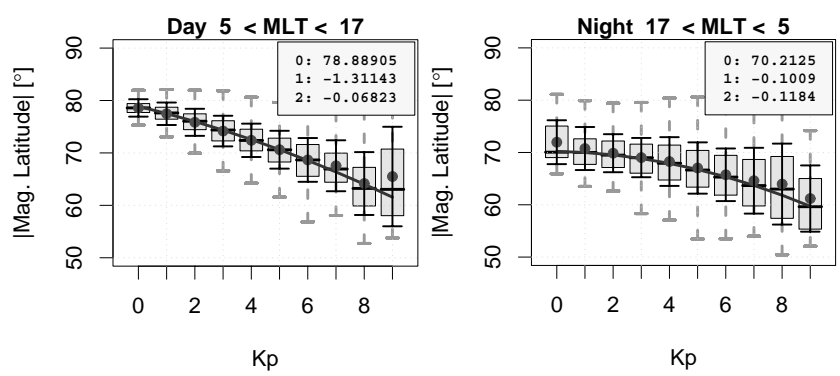

Fig. 10. Average latitude distribution of $50-\mathrm{Hz}$ current density peaks, $\mid j_{50 \mathrm{~Hz}}$,peak $\mid$, as a function of $K_{p}$ index. The format is the same as in Fig. 9.

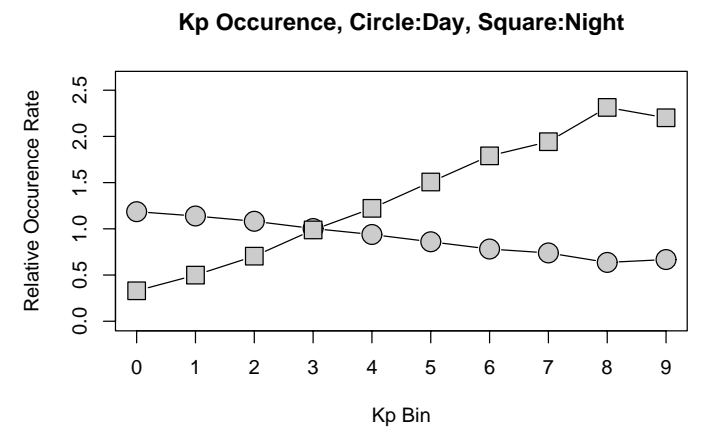

Fig. 11. Day and night occurrence frequencies of events in our table with respect to the all-day $K_{p}$ distribution.

$f_{i}^{x}=\left(N_{i}^{x} / \sum_{i=0}^{9} N_{i}^{x}\right) /\left(N_{i}^{a l l} / \sum_{i=0}^{9} N_{i}^{\text {all }}\right)$,

where $N_{i}^{x}$ is the count number in the $i$-th $K_{p}$ bin and with $x \in$ [day, night]. Dayside events occur preferentially during low activity. Relative to the all-days $K_{p}$ distribution the dayside population is slightly over-represented for $K_{p}<3$. Opposed to that, the nightside events occur preferentially during high activity. Their population is strongly over-represented for $K_{p}>3$.

We also examined the possible influence of solar activity on the small-scale FAC peak current densities. But neither a significant dependence on sunspot number nor on the solar flux index, F10.7, could be established.

Similarly, no clear influence was found of the solar zenith angle, $\chi$, on the current density peaks, $\left|j_{50 \mathrm{~Hz} \text {,peak }}\right|$. Figure 12 shows the corresponding dependence. It is practically flat for angles $\chi>45^{\circ}$. The increase for smaller angles is due to the fact that high $\left|j_{50 \mathrm{~Hz} \text {,peak }}\right|$ values are observed at lower latitudes during strongly disturbed conditions (see Fig. 10), and it is therefore not a genuine solar zenith angle effect. The independence of $\left|j_{50 \mathrm{~Hz} \text {,peak }}\right|$ on $\chi$ means that modifications in ionospheric conductivities due to solar illumination do not affect the KSFACs. This result is in contrast to the findings 

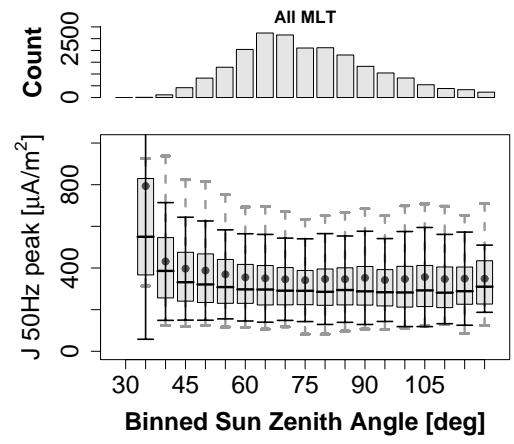

Fig. 12. Relationship of $50-\mathrm{Hz}$ current densities' peaks, $\left|j_{50 \mathrm{~Hz}, \text { peak }}\right|$, and solar zenith angle $\chi$. The format is the same as in Fig. 9.

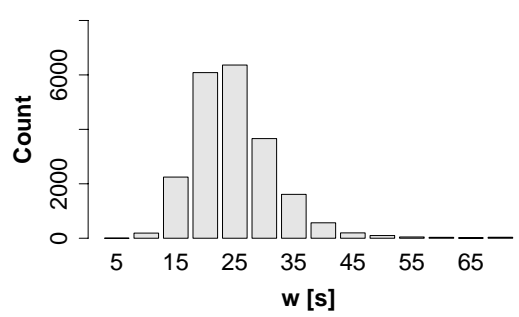

Fig. 13. Distribution of bursts width (2nd moment of temporal burst structure) in bins of $5 \mathrm{~s}$ ( $15 \mathrm{~s}$ correspond to $1^{\circ}$ in latitude).

of other authors (e.g. Fujii et al., 1981; Wang et al., 2005a) concerning the intensity of large-scale FACs.

\subsection{The shape of the current bursts (1-Hz data)}

In order to study the shape of the KSFAC bursts (latitude profile) in detail, we have to revert to the $1-\mathrm{Hz}$ data and the computed moments for the bursts, as described in Sect. 3. Figure 13 shows the distribution of the width calculated as the 2 nd moment of the temporal burst structure. The most frequent value of burst width is $25 \mathrm{~s}$, which corresponds to a length of $190 \mathrm{~km}$ or about $2^{\circ}$ in latitude when considering the satellite velocity of $7.6 \mathrm{~km} / \mathrm{s}$. The average width of all events is similar, $24.9 \mathrm{~s}$. The smallest width in our event list is $5.6 \mathrm{~s}$ $(43 \mathrm{~km})$ and the largest $101.3 \mathrm{~s}(764 \mathrm{~km})$, which corresponds to about $7^{\circ}$ in latitude. There is no significant dependence of the width on MLT, therefore we did not separate the width distribution for day- and nighttime.

The width of KSFAC bursts depends slightly on $K_{p}$, as shown in Fig. 13. During daytime the dependence is only very weak, but it is strong during nighttime. The regression line fitted to the median increases from $15 \mathrm{~s}$ at $K_{p}=0$ to almost $30 \mathrm{~s}$ at $K_{p}=9$. The increase in width on the nightside is probably again due to the widening of the auroral oval during disturbed conditions. In addition, it was shown by Wang
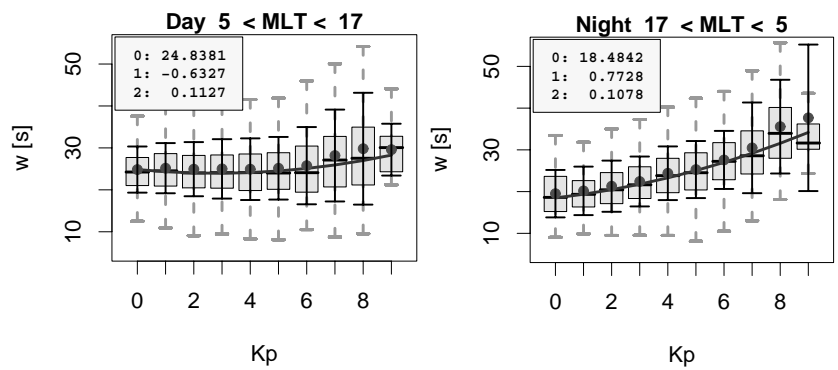

Fig. 14. Width of bursts as a function of $K_{p}$. Same format as in Fig. 9.

et al. (2006) that multiple FAC sheets form on the nightside during intense storms.

4.3 Relation between small-scale and large-scale current FACs

A question of relevance to this study is the relation of the KSFACs to the large-scale FAC patterns. A link between these two current types would be an important fact in the interpretation of source mechanisms. For this investigation we applied a superposed epoch analysis (SPE). We considered for the KSFACs the 1-Hz data and used the time of the current density peak as the key time for stacking the events (c.f. Fig. 4). For the large-scale FACs we took current estimates, low-pass filtered with a cutoff of $20 \mathrm{~s}$, corresponding to a minimum wavelength of $150 \mathrm{~km}$. The method for calculating the FAC current density from CHAMP data is described, for example, in Ritter and Lühr (2006).

When considering all KSFAC events from our list in a single analysis no persistent signature emerged. Therefore we applied the SPE separately to four different local time sectors. The obtained results are presented in Fig. 15. The choice of sectors takes into account both the average Region 1/Region 2 FAC distribution and the particular configurations around noon and midnight. Selected sector boundaries are 05:00-10:00 MLT (dayside dawn), 09:0013:00 MLT (noon = cusp region), 12:00-17:00 MLT (dayside dusk) and 21:00-02:00 MLT (midnight $=$ substorm region). The large-scale FACs density values were considered in two ways. First, we applied the SPE to current density estimates directly (taking into account the sign), and second, we calculated the absolute value and then stacked the current amplitude.

Average latitude profiles of the KSFACs (top panel in each frame) are compared with collocated FACs, when considering the currents' direction (positive for upward current) (middle panels) and when stacking the currents' amplitude (bottom panels). The SPE reveals a number of common features but also some differences between the chosen MLT sectors. As expected, the KSFAC exhibit quite a prominent peak of $\overline{j_{1 \mathrm{~Hz}}} \approx 100 \mu \mathrm{A} / \mathrm{m}^{2}$ at the key time. Current densities drop off 
quite rapidly on both sides of the central location until $\overline{j_{1 \mathrm{~Hz}}}$ reaches $40 \mu \mathrm{A} / \mathrm{m}^{2}$. Thereafter, the decay is more gradual.

The large-scale FAC amplitude (bottom panels) also shows a peak at the central location in all cases. The peak amplitude ranges around $2-2.5 \mu \mathrm{A} / \mathrm{m}^{2}$. Markedly different results are obtained in the middle panels where the current direction is taken into account. In the morning sector the KSFACs are predominately accompanied by downward FACs and in the afternoon sector by upward FACs. This implies that KSFACs occur preferably in connection with Region 1 FACs. In the noon (cusp) region no clear preference emerges for any of the FAC directions. Finally, around midnight, upward FACs are clearly dominant at the central location, but they are flanked both at the equatorward and poleward sides by downward currents. This signature resembles, in a way, the FAC structure associated with a westward travelling surge during a substorm break-up (e.g. Wang et al., 2005b).

We have identified the association of KSFACs to certain FAC regions in the different time sectors. There are, of course, exceptions from these average assignments. This can be deduced from the amplitude differences between the peaks in the middle and bottom panels. About $75 \%$ of the events fall into the described category. In the remaining cases KSFAC events are associated with regions of oppositely directed FACs.

\section{Discussion and conclusions}

In the previous sections we presented a comprehensive statistical analysis of kilometer-scale field-aligned currents. The large number of events $(\approx 20000)$ and the continuous sampling over 5 years allows one to address a number of different aspects of this phenomenon.

We have treated different features of the KSFACs separately, one is the peak current density within a burst, others are an occurrence statistics and the properties of the bursts. The rational for relying on the full resolution $50-\mathrm{Hz}$ data for the first one is to present the extreme cases in a form processed as little as possible. For the description of the other characteristics we made use of the $1-\mathrm{Hz}$ envelop data. These data reflect the average properties of the bursts sufficiently well. Due to the small transverse scale size of the individual current filaments $(\approx 1 \mathrm{~km})$, only a small subset of KSFAC structures are encountered by CHAMP, and the probability for hitting the strongest filament in a burst is not high. For that reason our selection of $\left|j_{50 \mathrm{~Hz} \text {,peak }}\right|$ has to be regarded as a lower bound. The 1-Hz data may thus be more representative for the average properties. Due to the high correlation between the peak amplitudes (c.f. Fig. 6), we are encouraged to use both data sets equivalently.

One of the most obvious results of our analysis is the concentration of events at high latitudes. They are clearly related to the auroral current systems and in particular to the cusp region, where we find the highest occurrence rates (c.f. Fig. 8).

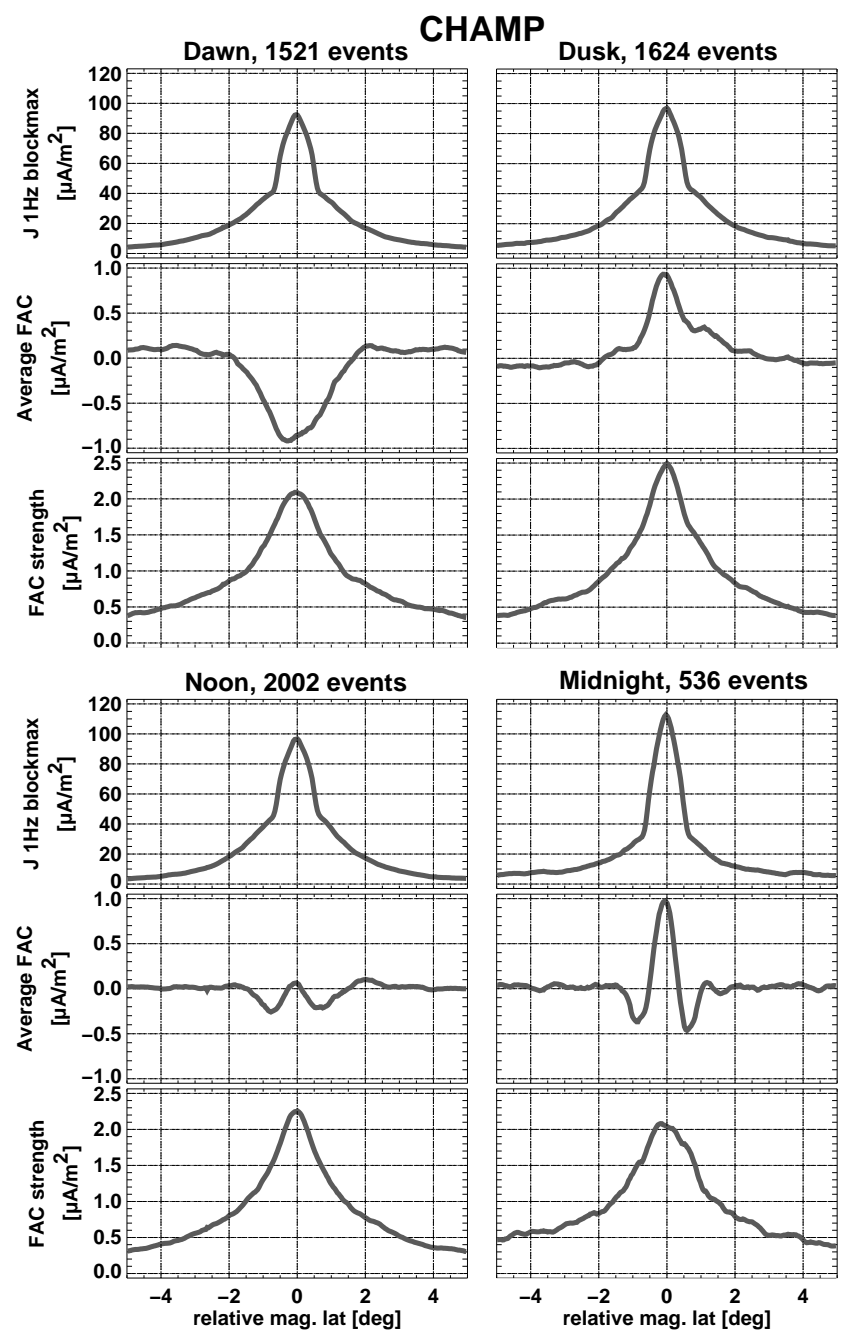

Fig. 15. Superposed Epoch Analysis: relative location of KSFAC bursts with respect to large-scale field-aligned currents for four different MLT intervals. 05:00-10:00 MLT (dayside dawn), 09:0013:00 MLT (noon = cusp region), 12:00-17:00 MLT (dayside dusk, upper right) and 21:00-02:00 MLT (midnight = substorm region).

Supporting evidence for the connection to the auroral oval comes from the equatorward displacement of the event locations during times of higher $K_{p}$ values (c.f. Fig. 10). The different behaviours on the day- and nightsides reflect quite closely the well-known polar cap expansion characteristics during periods of high magnetic activity.

When accepting the established relation of KSFACs to the large-scale auroral currents (Sect. 4.3), we have to answer a challenging question, namely why is there almost no dependence of the peak current density on geophysical conditions? Within the $K_{p}=9$ bin we observe that $\mid j_{50 \mathrm{~Hz} \text {,peak } \mid}$ amplitudes stretching from 200 to $3311 \mu \mathrm{A} / \mathrm{m}^{2}$. On the dayside we still find some increase in the average current density by about a factor of 1.6 from $K_{p} 0$ to 7 (c.f. Fig. 9), but on 
the nightside the increase over the same $K_{p}$ range is already marginal. Apart from that there is practically no dependence of $\left|j_{50 \mathrm{~Hz} \text {,peak }}\right|$ on any of the other parameters, like EUV solar flux (F10.7), solar zenith angle, or seasons, which are wellknown to control auroral currents strengths (e.g. Peria et al., 2000; Wang et al., 2005a).

On the other hand, it is interesting to note that the occurrence frequency of KSFACs shows clear dependencies on the geophysical conditions (c.f. Fig. 5). Rather pronounced is the difference between summer and winter seasons, but also the solar cycle effect is as expected for auroral currents. Both observations can be reconciled when we assume a kind of switch-on, switch-off effect for the KSFACs. It is probably required that the intensity of the associated large-scale FAC surpasses a certain threshold level, for the KSFACs to occur. If once initiated, then the current density of the KSFACs seems to be controlled by other factors other than the geophysical conditions. Our proposed explanation of KSFACs in terms of Alfvénic structures is also supported by such a switch-on, switch-off scenario (see below).

Further supporting evidence for the switch-on scenario can be drawn from the KSFAC occurrence statistics. It is known that there is almost no correlation between the intensity of FACs on the dayside and $K_{p}$ (e.g. Ritter et al., 2004), but the substorm activity on the nightside is better reflected by $K_{p}$ values. Similarly, for the seasonal dependence, it has been shown, for example, by Fujii et al. (1981) and Wang et al. (2005a) that FACs on the dayside exhibit a strong seasonal dependence of their intensity. As expected, strong FACs occur in the sunlit (summer) hemisphere. Conversely, the nightside FAC intensities show only a weak seasonal dependence. Both of these features of FAC intensity are nicely reflected in the KSFAC occurrence frequencies (c.f. Fig. 5a). Along the same line, the solar cycle dependence of the occurrence rate can be explained by changes in auroral current strength (c.f. Fig. 5b).

In the quest for the generation mechanism of our smallscale FACs we will take a closer look at the relation to the large-scale FAC pattern around the auroral oval. Important results have been revealed by the superposed epoch analysis, SPE. The local time sectors for the analysis were deliberately chosen to help identifying a possible connection to the Iijima-Potemra pattern. A common feature in all sectors is the collocation of KSFAC bursts with large-scale FAC peaks. Interestingly, the average amplitude of the large-scale FAC (Fig. 15, bottom panels) is quite similar in all sectors, varying between 2 to $2.5 \mu \mathrm{A} / \mathrm{m}^{2}$. This seems to reflect the current density needed to switch on the KSFACs, since $\overline{j_{1} \mathrm{~Hz}}$ reaches, on average, similar peak values in all considered time zones. Quite impressive is the large amplitude ratio between the two FAC types. When taking into account that the unsmoothed peak current density is 3.7 times larger (c.f. Fig. 6), we obtain a ratio of more than 150 .

Significant differences between the time sectors emerge when looking at the middle panels (c.f. Fig. 15), where the average FAC direction is plotted. The opposite direction of current flow, downward at pre-noon and upward at postnoon, implies that KSFACs are preferably generated in connection with Region 1 FACs. However, some $25 \%$ of the events coincide with Region 2 currents. Around the cusp region there is no preference for upward or downward currents. This is expected, since the direction of the prominent FACs around noon is determined largely by the orientation of the IMF $B_{y}$ component. In case of the night sector the curve in the middle panel shows the typical FACs pattern common for substorm onsets (Wang et al., 2005b). This feature suggests a close relation of the nightside KSFACs to the substorm expansion phase.

Another feature common in all time sectors (Fig. 15) is the average latitude profile of the KSFAC density, $\overline{j_{1 \mathrm{~Hz}}}$. We find a rather narrow current density distribution for values above $40 \mu \mathrm{A} / \mathrm{m}^{2}$, confined to a latitude range of only $1.5^{\circ}$. Beyond that the decay is much more gradual. In Sect. 4.2 we investigated the shape of the bursts. The distribution of the bursts' width peaks at $25 \mathrm{~s}$ (c.f. Fig. 13), which corresponds well to the confined shape of $\overline{j_{1} \mathrm{~Hz}}$ in Fig. 15 $\left(25 \mathrm{~s} \times 7.6 \mathrm{~km} / \mathrm{s}=175 \mathrm{~km}=1.6^{\circ}\right)$. Necessary conditions for the existence of large amplitude KSFACs seem to exist only in the vicinity of strong background FACs.

We suggest that the intense KSFACs can be interpreted as trapped small-scale Alfvénic structures in a resonator. On the basis of our results we cannot decide which type of resonator is involved, the common IAR (Trakhtengerts and Feldstein, 1991; Lysak, 1991) or the RAAR (Pilipenko et al., 2002). They differ mainly in the height and nature of the upper boundary, as well as the plasma instabilities involved. Both resonators have properties which are in agreement with our results, as outlined in the following.

Regarding the common IAR, Trakhtengerts and Feldstein (1991) and Lysak (1991) have shown that the small-scale Alfvénic waves trapped in the resonator are excited if the convection electric field exceeds a certain threshold. This could explain our switch-on scenario, mentioned above. Since the convection electric field and the large-scale FACs are clearly related, this would also support our observed relationship between the large-scale FACs and the KSFACs (Fig. 15). The limiting magnitude of the KSFACs, and thus the switch-off mechanism, can also be understood in terms of the Trakhtengerts and Feldstein (1991) theory. A saturation of the involved IAR instability occurs when anomalous resistance is generated and thus the upper boundary of the IAR is changed. Furthermore, Trakhtengerts and Feldstein (1991) established that ionospheric conductance and the threshold of the IAR instability are inversely related, i.e. the lower the conductance, the lower the instability threshold is due to a decreased damping. This could explain our observed seasonal dependence of the KSFAC occurrence frequency (Fig. 5).

Regarding the RAAR, a nonlinear wave particle interaction is considered important for the formation of the AAR. 
For its initiation strong overcritical field-aligned current densities are required (Haerendel, 1990). The trapping of wave power is particularly efficient for Alfvén waves with small transverse scales (Vogt, 2002). Pilipenko et al. (2002) estimated that the Q-factor of their RAAR increases from values below 1 at transverse scales of several $100 \mathrm{~km}$ to $Q>10$ for $\mathrm{km}$-size transverse structures. All these criteria are fulfilled by our observations. The authors state, however, that Alfvén waves below a certain spatial scale are strongly damped upon reflection at the conductive E-layer. This is consistent with the spectral decay for wavelength below $1 \mathrm{~km}$ (Fig. 3).

The presence of a KSFAC burst, for example, implies that an auroral acceleration region exists along the corresponding field line. This necessary condition can well account for the switch-on, switch-off characteristic of the FAC bursts. Once a resonator is set up, the achieved wave amplitudes are controlled by the quality factor of the system, not by the geophysical conditions. According to Pilipenko et al. (2002), the Q-factor of the RAAR depends on the wave dissipation rate in the ionosphere and on the reflection features of the AAR. Although the former depends on ionospheric conductivity and thus on geophysical conditions, the influence of the AAR reflection properties dominates for $\mathrm{km}$-size transverse structures. This independence of the observed wave amplitudes is also in line with the observations (our $\left|j_{50 \mathrm{~Hz} \text {,peak }}\right|$ ). We find for all levels of magnetic activity that KSFAC peak current densities are more than 100 times stronger than that of the accompanying large-scale FACs. This implies very efficient reflectors on both sides of the resonator. As a consequence, the Joule heating associated with a KSFAC burst must be low. Since the E-layer is a conducting reflector, the magnetic wave field of the upward and downward propagating Alfvén waves add up, maximising just above the $\mathrm{E}$ region. This is well in line with the large observed amplitudes at CHAMP's low orbit. It is thus no surprise that the peak current densities presented here are higher than anything reported earlier. The electric field of these waves should practically vanish at these low heights, in order to keep the losses low. But as CHAMP does not perform reliable E-field measurements, we have no observational support for this vanishing.

Our results also support the existence of a density cavity which is important for both resonators. The magnetic signature shown in Fig. 1 can be interpreted in terms of a pair of up- and down-going currents (e.g. Dubinin et al., 1985; Boehm et al., 1990; Stasiewicz et al., 1997). Mishin and Förster (1995) have shown that such a pair of medium-scale FACs is the lowest eigenmode of a transverse Alfvén waveguide, i.e. a density depletion across the magnetic field. The presence of such a waveguide is also crucial for the IAR instability.

Based on Akebono data Hirano et al. (2005) report that Alfvén waves with small transverse scales are commonly accompanied by high fluxes of low-energy $(<100 \mathrm{eV})$ electrons. This is consistent with Freja and FAST observations
(Stasiewicz et al., 2000; Chaston et al., 2002). We have no particle measurements on CHAMP, but from the association of the KSFAC events to certain auroral regions we can make some inferences. The highest occurrence rates are encountered in the cusp. This region is characterised by its high flux of low energy electrons (Newell and Meng, 1992). On the dayside part of the flanks we find a concentration of the events at Region 1 latitudes. These regions are also populated significantly by lower energy electrons. The nightside flanks are almost void of KSFAC events (c.f. Fig. 8). Here the inverted $V$ type of precipitation is more common. The high energetic electrons dominating here are obviously not so efficient in setting up a reflector within the AAR of the bottom side magnetosphere. (Note that high energy particles are also created, according to the Trakhtengerts and Feldstein, 1991, theory.) Finally, the events around midnight are associated with substorm break-ups. Here again, an appreciable amount of low energy electrons can be found in the upward FAC connected to the surge head.

Here we have suggested the Alfvén wave resonator as a possible driver for the observed intense KSFACs. There may well be other, more appropriate generation mechanisms. A critical assessment of the predictions from the Alfvén resonator model with the KSFAC characteristics will be the subject of another dedicated study.

In summary, we have presented a comprehensive survey of a distinct class of kilometer-scale FAC structures. The magnetic field signatures have been related to Alfvén waves trapped in a recently proposed Alfvén resonator (RAAR). Our observed large current density peaks may reflect the high quality of the resonator for the small wavelength. Our results about KSFACs may represent the global and temporal occurrence of this resonator and its relation to geophysical conditions. Furthermore, its relation to the various FAC regions could be established. The background field-aligned currents have to fulfill certain conditions to switch on the resonator. The amplitude of the resonant wave is determined by the performance of the reflectors, not by geophysical conditions. In a follow-on study it is planned to perform a quantitative comparison of the observed wave features with the theoretical predictions for the different Alfvén wave resonators.

Acknowledgements. We thank P. Ritter for providing the large-scale CHAMP FAC estimates. Fruitful discussions with S. Buchert are highly acknowledged. The operational support of the CHAMP mission by the German Aerospace Center (DLR) and the financial support for the data processing by the Federal ministry of Education and Research (BMBF) are gratefully acknowledged. Some figures are created with the statistical software R (R Development Core Team, 2006).

Topical Editor M. Pinnock thanks E. Mishin and another anonymous referee for their help in evaluating this paper. 


\section{References}

Belyaev, P. P., Bösinger, T., Isaev, S. V., Trakhtengerts, V. Y., and Kangas, J.: First evidence at high latitudes for the ionospheric Alfvén resonator, J. Geophys. Res., 104, 4305-4318, 1999.

Boehm, M. H., Carlson, C. W., McFadden, J. P., Clemmons, J. H., and Mozer, F. S.: High resolution sounding rocket observations of large amplitude Alfvén waves, J. Geophys. Res., 95, 12 157$12171,1990$.

Boudouridis, A. and, E. Z., Lyons, R., Anderson, P. C., and Lummerzheim., D.: Effect of solar wind pressure pulses on the size and strength of the auroral oval, J. Geophys. Res., 108, 8012, doi:10.1029/2002JA009373, 2003.

Burch, J. L., Reiff, P. H., and Sugiura, M.: Upward electron beams measured by DE-1 - A primary source of dayside region-1 Birkeland currents, Geophys. Res. Lett., 10, 753-756, 1983.

Chaston, C. C., Bonnell, J. W., Carlson, C. W., Berthomier, M., Peticolas, L. M., Roth, I., McFadden, J. P., Ergun, R. E., and Strangeway, R. J.: Electron acceleration in the ionospheric Alfven resonator, J. Geophys. Res., 107, 1413, doi:10.1029/ 2002JA009272, 2002.

Chmyrev, V., Oraevsky, V. N., Bilichenko, S. V., Isaev, N. V., Stanev, G. A., Teodosiev, D. K., and Shkolnikova, S. I.: The fine structure of intensive small-scale electric and magnetic fields in the high-latitude ionosphere as observed by IntercosmosBulgaria 1300 satellite, Planet. Space Sci., 33, 1383-1388, 1985.

Dubinin, E. M., Israelevich, P. L., Nikolaeva, N. S., Kutiev, I., and Podgorny, I. M.: Localized auroral disturbance in the morning sector of topside ionosphere as a standing electromagnetic wave, Planet. Space Sci., 33, 597-606, 1985.

Fujii, R., Iijima, T., Potemra, T. A., and Sugiura, M.: Seasonal dependence of large-scale Birkeland currents, Geophys. Res. Lett., 8, 1103-1106, 1981.

Fukunishi, H., Fujii, R., Kokubun, S., Tohyama, F., and Mukai, T.: Small-scale field-aligned currents observed by the Akebono (EXOS-D) satellite, Geophys. Res. Lett., 18, 297-300, 1991.

Golovchanskaya, I. V., Ostapenko, A., and Kozelov, B.: Relationship between the high-latitude electric and magnetic turbulence and the Birkeland field-aligned currents, J. Geophys. Res., 111, A12 301, doi:10.1029/2006JA011835, 2006.

Haerendel, G.: Field-aligned currents in the Earth's magnetosphere, in: Physics of Magnetic Flux Ropes, edited by: Russell, C., Priest, E., and Lee, L., pp. 539-553, AGU, Washington, 1990.

Hirano, Y., Fukunishi, H., Kataoka, R., Hasunuma, T., Nagatsuma, T., Miyake, W., and Matsuoka, A.: Evidence for the resonator of inertial Alfvén waves in the cusp topside ionosphere, J. Geophys. Res., 110, A07 218, doi:10.1029/2003JA010329, 2005.

Iijima, T. and Potemra, T.: Field-aligned currents in the dayside cusp observed by Triad, J. Geophys. Res., 81, 5971-5979, 1976.

Lühr, H., Oelschlägel, W., Rinnert, K., and Wilhelm, K.: Observations of field-aligned current structures during CEASAR flight II, vol. 350 of SP-270, ESA, 1987.

Lühr, H., Warnecke, J., Zanetti, L. J., Lindqvist, P. A., and Hughes, T. J.: Fine structure of field-aligned current sheets deduced from spacecraft and ground-based observations: Initial FREJA results, Geophys. Res. Lett., 21, 1883-1886, 1994.

Lühr, H., Warnecke, J., and Rother, M.: An algorithm for estimating field-aligned currents from single spacecreaft magnetic field measurements: A diagnostic tool applied to Freja satellite data, IEEE Trans. Geosci.-Remote Sens., 34, 1369-1376, doi:
10.1109/36.544560, 1996.

Lysak, R. L.: Feedback instability of the ionospheric resonant cavity, J. Geophys. Res., 96, 1553-1568, doi:10.1029/90JA02154, 1991.

Mishin, E. and Förster, M.: 'Alfvénic shocks' and low-altitude auroral acceleration, Geophys. Res. Lett., 22, 1745-1748, 1995.

Neubert, T. and Christiansen, F.: Small-scale, field-aligned currents at the top-side ionosphere, Geophys. Res. Lett., 30, 2010, doi: 10.1029/2003GL017808, 2003.

Newell, P. T. and Meng, C.-I.: Mapping the dayside ionosphere to the magnetosphere according to particle precipitation characteristics, Geophys. Res. Lett., 19, 609-612, doi:10.1029/ 92GL00404, 1992.

Peria, W., Carlson, C., Ergun, R., McFadden, J., Bonnell, J., Elphic, R., and Strangeway, R.: Characteristics of Field-Aligned Currents Near the Auroral Acceleration Region: FAST Observations, in: Magnetospheric Current Systems, edited by: Shin-ichi, O., Fujii, R., Hesse, M., and Lysak, R. L., no. 118 in Geophysical Monograph, p. 181, American Geophysical Union, Washington, D.C., 2000.

Pilipenko, V. A., Fedorov, E. N., and Engebretson, M. J.: Alfvén resonator in the topside ionosphere beneath the auroral acceleration region, J. Geophys. Res., 107, 1257, doi:10.1029/ 2002JA009282, 2002.

Potemra, T. A., Zanetti, L. J., Erlandson, R. E., Bythrow, P. F., Gustafsson, G., and Acuna, M. H.: Observations of large-scale Birkeland currents with Viking, Geophys. Res. Lett., 14, 419422, 1987.

R Development Core Team: R: A Language and Environment for Statistical Computing, R Foundation for Statistical Computing, Vienna, Austria, http://www.R-project.org, ISBN 3-900051-070, 2006.

Reigber, C., Lühr, H., and Schwintzer, P.: CHAMP mission status, Adv. Space Res., 30, 129-134, 2002.

Ritter, P. and Lühr, H.: Curl-B technique applied to Swarm constellation for determining field-aligned currents, Earth Planets Space, 58, 463-476, 2006.

Ritter, P., Lühr, H., Maus, S., and Viljanen, A.: High latiutude ionospheric currents during very quiet times: their characteristics and predictabilities, Ann. Geophys., 22, 2001-2014, 2004, http://www.ann-geophys.net/22/2001/2004/.

Russell, C. and McPherron, R.: Semiannual variation of geomagnetic activity, J. Geophys. Res., 78, 92-108, 1973.

Stasiewicz, K. and Potemra, T.: Multiscale current structures observed by Freja, J. Geophys. Res., 103, 4315-4325, 1998.

Stasiewicz, K., Gustafsson, G., Marklund, G., Lindqvist, P.-A., Clemmons, J., and Zanetti, L.: Cavity resonators and Alfvén resonance cones observed on Freja, J. Geophys. Res., 102, 25652576, 1997.

Stasiewicz, K., Bellan, P., Chaston, C., Kletzing, C., Lysak, R., Maggs, J., Pokhotelov, O., Seyler, C., Shukla, P., Stenflo, L., Streltsov, A., and Wahlund, J.-E.: Small Scale Alfvénic Structure in the Aurora, Space Sci. Rev., 92, 423-533, doi:10.1023/A: 1005207202143, 2000.

Trakhtengerts, V. Y. and Feldstein, A. Y.: Turbulent Alfven boundary layer in the polar ionosphere 1. Excitation conditionsand energetics, J. Geophys. Res., 96, 19363-19374, 1991.

Trakhtengertz, V. Y. and Feldstein, A. Y.: Quiet auroral arcs: Ionosphere effect of magnetospheric convection stratification, Planet. 
Space Sci., 32, 127-134, 1984.

Vogt, J.: Alfvén wave coupling in the auroral current circuit, Surv. Geophys., 23, 335-377, 2002.

Vogt, J. and Haerendel, G.: Reflection and transmission of Alfvén waves at the auroral acceleration region, Geophys. Res. Lett., 25, 277-280, doi:10.1029/97GL53714, 1998.

Wang, H., Lühr, H., and Ma, S.-Y.: Solar zenith angle and merging electric field control of field-aligned currents: A statistical study of the southern hemisphere, J. Geophys. Res., 110, A03 306, doi: 10.1029/2004JA010530, 2005a.
Wang, H., Lühr, H., Ma, S.-Y., and Ritter, P.: Statistical study of the substorm onset: its dependence on solar wind parameters and solar illumination, Ann. Geophys., 23, 2069-2079, 2005b.

Wang, H., Lühr, H., Ma, S. Y., Weygand, J., Skoug, R. M., and Yin, F.: Field-aligned currents observed by CHAMP during the intense 2003 geomagnetic storm events, Ann. Geophys., 24, $311-$ 324, 2006,

http://www.ann-geophys.net/24/311/2006/. 